\title{
DYNAMICS OF THICK SPIRALS
}

\author{
P.A. PATSIS AND P. GROSB $\emptyset \mathrm{L}$ \\ ESO, Karl Schwarzschild Str. 2, D-85748, Garching, Germany
}

\section{Introduction}

Most studies of spiral structure in galaxies use 2D models, since the motion of the stars is supposed to be confined very close to the plane of symmetry. However, galactic disks have a finite thickness and the vertical variation of spiral perturbations should be considered. The effect of 3D orbits in the dynamics of normal spiral galaxies is examined in this paper. We present the basic orbital behavior of the families of periodic orbits, which support a thick spiral pattern.

\section{The potential}

The potential used for the calculations has the form: $\Phi=\Phi_{d}+\Phi_{h}+\Phi_{s}$, where the axisymmetric part consists of a Miyamoto disk $\Phi_{d}$ (Miyamoto and Nagai 1975) and a halo:

$$
\Phi_{h}(r, z)=\frac{v_{h}^{2}}{2} \ln \left(1+\frac{1}{r_{c}^{2}}\left(r^{2}+z^{2}\right)\right)
$$

The constants $v_{h}$ and $r_{c}$ are the maximum rotational velocity and the core radius of the halo respectively. The spiral perturbation is:

$$
\Phi_{s}(r, \theta, z)=A r \exp \left(-\epsilon_{s} r\right)\left[\cos \left(2\left(\frac{\ln r}{\tan i}\right)-2 \theta\right)\right] \operatorname{sech}^{2}\left(\frac{z}{z_{0}}\right),
$$

where $A$ is the amplitude of the perturbation, $\epsilon_{s}$ is the inverse radial scale length of the spiral, $i$ is its pitch angle and $z_{0}$ its scale height. We have calculated the orbits in a frame rotating around the z-axis. The angular pattern speed was taken so that corotation was located close to the $R_{\max }$ distance of van der Kruit and Searle (1982). The parameters used for the axisymmetric part attempted to fit the profiles proposed by van der Kruit and 
Searle (1982) (Model A) and by Barnaby and Thronson (1994) (Model B) for the surface photometry of NGC 5907. We added a strong open spiral with $i=30^{\circ}$.

\section{Conclusions}

The main conclusions for the 3D models are:

- A thick spiral pattern is supported by 3D families of periodic orbits with elliptical projections on the equatorial plane. These families are bifurcations of the 2D central periodic family at the vertical resonances of the model.

- The structure of 3D periodic orbits makes it difficult to create selfconsistent models of spirals reaching corotation.

We found two different types of orbital behavior in the examined cases corresponding to the two different models of the photometry of NGC 5907.

- In Model $A$ the successive 3D families which support the pattern are producing a "stair-type" edge-on profile. The most important 3D families of periodic orbits are bifurcated at the vertical $2 / 1,3 / 1$ and $4 / 1$ resonances. This way it is possible for peanut shaped structures to appear in the central regions of the response model without invoking any bar in the imposed potential.

- In Model $B$ the 3D families are characterized by similar mean $\mathrm{z}$ values of their orbits. Thus no "stair-type" effect appears. The most important families of $3 \mathrm{D}$ periodic orbits are bifurcated at the vertical $4 / 1$, $5 / 1$ and $6 / 1$ resonances. Their projections on the equatorial plane are identical with the central family even after several kpc beyond the bifurcating point.

The above differences reflect the different locations of the vertical resonances in the models (Patsis and Grosbøl 1995).

\section{Acknowledgements}

This work was supported by the program "Human Capital and Mobility" of the EU, through the contract CHRA-CT93-0330.

\section{References}

Barnaby D., Thronson H.A.Jr, 1994, A J 107, 1717

Miyamoto M., Nagai R., 1975 PASJ 27, 533

Patsis P.A., Grosbøl P., 1995 - A\&A submitted

van der Kruit P.C., Searle L., 1982 A\&A 110, 61 